\title{
Power Ambulance System for Energy Harvested Wireless Sensor Network
}

\author{
Amit Kumar Bindal \\ PhD. Research Scholar \\ I.T. Department \\ M.M.University \\ Mullana, Haryana India
}

\author{
Anuj Jain \\ Assistant Professor \\ C.S.E. Department \\ M.M.University \\ Mullana, Haryana India \\ R. B. Patel,Ph.D \\ Professor \\ C.S.E. Department \\ Chandigarh College \\ Engineering \\ Chandigarh, India
}

\author{
Devendra Prasad, Ph.D \\ Professor \& Head \\ I.T. Department \\ M.M.University \\ Mullana, Haryana India
}

\begin{abstract}
Wireless sensor networks (WSNs) consist of a number of sensor nodes, which are able to collect information from the environment by measuring diverse environment variables, like temperature, humidity, sound etc. Sometimes the sensor nodes (SN) are expected to be deployed in unattended environments for long durations; the major challenge is tapping on ambient energy to power such networks. This paper investigates on providing dedicated power supply to all static sensor nodes (SN) through mobile power nodes (MPNs); which is equipped with solar cells and super capacitors to store harvested energy. The MPNs are used to supply energy to all other static sensor nodes which has energy below minimum threshold level. The super capacitor guarantees a longer lifetime in terms of charge cycles, it presents itself as a "green" technology compared to batteries and it has a wide range of operating temperature.
\end{abstract}

Keywords: Mobile Power Nodes (MPN), Sensor Nodes (SN), Super Capacitor

\section{INTRODUCTION}

Often WSNs are deployed in regions that are difficult to access and so the sensor nodes should not require any maintenance at all under ideal condition. They must be energetically autonomous and independent. This implies that once the batteries/super capacitors are installed for the sensor nodes, they do not need to be replaced or recharged for a long period of time and really operate in an autonomous manner for life-long operation.

Collections of tiny, inexpensive wireless sensor nodes (modules), organized in clusters and networks deployed over a geographical area, capable to integrate continuous and unobtrusive measurement, computing and wireless communication, have attracted much attention during the last decade in forming the concept of smart spaces. Batteries provide the most obvious power source of sensor nodes. In spite of the fact that battery technology is mature, extensively commercialized, and completely self-contained, even for relatively large battery capacity and moderate communication traffic requirements, the mean time to replacement or recharging is only two or three years. Several solutions to the power problem exist, such as reducing power consumption to the point where batteries can elongate the sensor module's lifetime [6]. Another solution is energy harvesting-EH (or energy scavenging) - that is extracting energy from ambient sources. The harvesting circuit must stay in active mode permanently, to be ready to capture harvestable energy whenever it becomes available, and to be capable to provide an output as the application requires. The power consumption of the harvester has to be very small so that the energy consumed by this circuit is much smaller than the energy provided by the ambient sources. The second key component of the harvester is its high energy retention, i.e. the capability to store the captured energy for as long as possible with minimal leakage or loss. The energy harvesting circuit must also economize the stored energy in order to provide correct operation for the intended application.

\section{LITERATURE SURVEY}

An emerging technique that promises to circumvent this limitation is environmental energy harvesting (scavenging). The process of extracting energy from the surrounding environment and converting it into consumable electrical energy is termed as energy harvesting or power scavenging [1]. In general, harvesting sources are used to increase the lifetime and capability of SNs by augmenting the battery usage. Energy harvesting is most applicable to applications that demand small amounts of continuous power or that have short periods of high-power use, which previously harvested and stored energy can provide for. SNs are typical candidate devices for such applications.

There are many types of energy harvesters each offering differing degrees of usefulness depending on the application [3] and the area of deployment [11]. The various sources for energy harvesting are wind turbines, photovoltaic cells, human body, thermoelectric generators and mechanical vibration devices such as piezoelectric devices or electromagnetic devices [4].

Table 1 shows power outputs for typical energy scavenging devices [2]. The classification of energy harvesting can be organized on the basic of the form of energy they use to scavenge the power, and in general, we can distinguish three types of harvesting sources from surrounding [1].

Table 1: Power output from various energy scavenging technologies [5]

\begin{tabular}{|l|l|}
\hline Harvesting Technology & Power Density \\
\hline Nuclear Reaction & $80 \mathrm{~mW} / \mathrm{cm}^{3}$ \\
& $1 \mathrm{E} 6 \mathrm{mWh} / \mathrm{cm}^{3}$ \\
\hline Solar Cell- Direct Sun & $15 \mathrm{~mW} / \mathrm{cm}^{2}$ \\
\hline Solar cell-Indoors & $0.006 \mathrm{~mW} / \mathrm{cm}^{2}$ \\
\hline Solar cell-Desk Lamp-60W & $0.57 \mathrm{~mW} / \mathrm{cm}^{2}$ \\
\hline
\end{tabular}




\begin{tabular}{|l|l|}
\hline Piezoelectric-shoe Inserts & $330 \mu \mathrm{W} / \mathrm{cm}^{2}$ \\
\hline Acoustic Noise-100 dB & $9.6-4 \mathrm{~mW} / \mathrm{cm}^{2}$ \\
\hline Passive human powered system & $1.8 \mathrm{~mW}$ \\
\hline
\end{tabular}

\section{Super Capacitors}

Super capacitor, also referred to as ultracapacitors or electrochemical double layer capacitors, is another electrochemical energy system. One reason is which is very scalable and its performance scales well with its size and weight. It can be identified that super capacitor has much higher peak power density than the other energy storage devices like batteries and fuel cells. This means that super capacitor can deliver more electrical power than batteries and fuel cells within a short time. The peak power densities of super capacitors are well above $1000 \mathrm{~W} / \mathrm{kg}$ level whereas the power densities of all types of batteries are in the range of 60 $\mathrm{W} / \mathrm{kg}$ to $200 \mathrm{~W} / \mathrm{kg}$ and fuel cells are well below $100 \mathrm{~W} / \mathrm{kg}$. Hence for burst power operation, supercapacitors are better choice than batteries and fuel cells. Supercapacitors, also referred to as ultracapacitors or electrochemical double layer capacitors, differ from the conventional electrostatic and electrolytic capacitors as they contain an electrolyte which enables the electrostatic charge to also be stored in the form of ions [9]. They utilize the larger surface area electrodes and thinner dielectrics to achieve greater capacitances [10]. Since these devices are designed to store energy using both ionic capacitance and surface redox reactions.

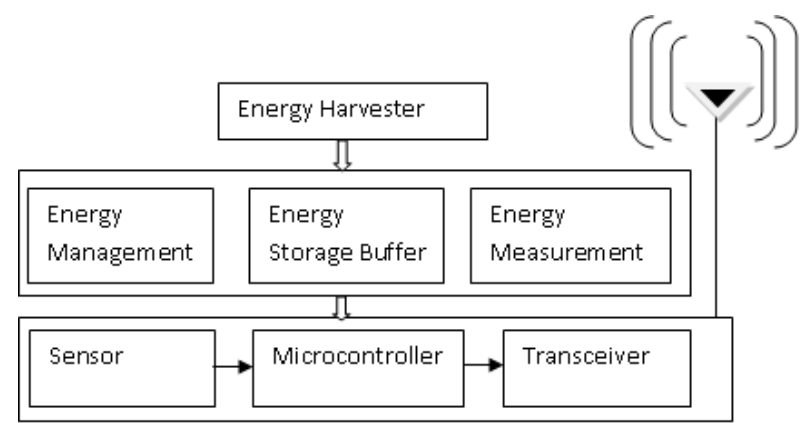

Figure 1 Block Diagram of a Typical Energy Harvesting Wireless Sensor Node

A typical energy harvesting wireless sensor node is depicted in blocks in Figure 1.The wireless sensor node consists of a microcontroller, a transceiver chip and a sensor depending on the application. The energy harvesting system consists of a harvester or harvesting device such as a solar panel, thermoelectric generator, cantilever beams etc. Depending on the type of harvester, the raw output of the harvester is processed using components such as rectifiers [8], DC-DC converters for stepping the voltage level up or down, maximum power point tracking (MPPT) devices and so on. The harvested power is stored in an energy storage buffer [7].

\section{SYSTEM MODEL}

A dedicated power management module is the heart of our network that pumps power throughout the system. This module monitors the health of the system at dynamic times and takes decisions for the healthy working of the system. Our System comprises of large number of Static Sensor Nodes (SN) which are homogeneous. The network is randomly deployed. Each SN has its unique id and is characterized by all the usual limitations in a WSN. The static sensor node uses its unique id for communication of data. The Base station (BS) maintains a table representing the unique id \& corresponding energy factor index of each node.

The network also comprises of Mobile Power Nodes (MPN) which are capable of energy harvesting. MPN Uses super capacitor to store harvested energy; which can hold charge for a long time. The main focus of MPNs is used to charge the static sensor nodes. They are randomly deployed in few numbers (Decided by the MPN factor $\mathrm{m}$ ). These nodes are equipped with high communication (transmission) range. All MPNs can communicate to the BS directly or through other MPNs. Base Station (BS) will maintain the location of all Mobile Power Nodes. In case of node failure or loss of charge by a SN the closest power node will move to a critical sensor node $\&$ charge the node which will be decided by BS in terms of distance between them. When no sensor node is in critical state then, nodes which are approaching towards critical stage will be charged first. Healthy nodes are not likely to obtain any energy aid.

The locations of all static sensor nodes are identified and maintained by BS. Base station (BS) maintains a table representing the unique id \& corresponding energy level of each static sensor node. Once a node falls into Critical(C) category; the node will be put into the queue maintained by the BS. BS will instruct the closest MPN to charge the critical node. After the critical node gets charged, either the MPN or critical node will send the acknowledgement to the BS, so that the node will be removed from the queue. If a MPN cannot attend a critical node which needs charging, then an error message will be sent to the BS. Base Station (BS) also maintains the location of all Mobile Power Nodes.

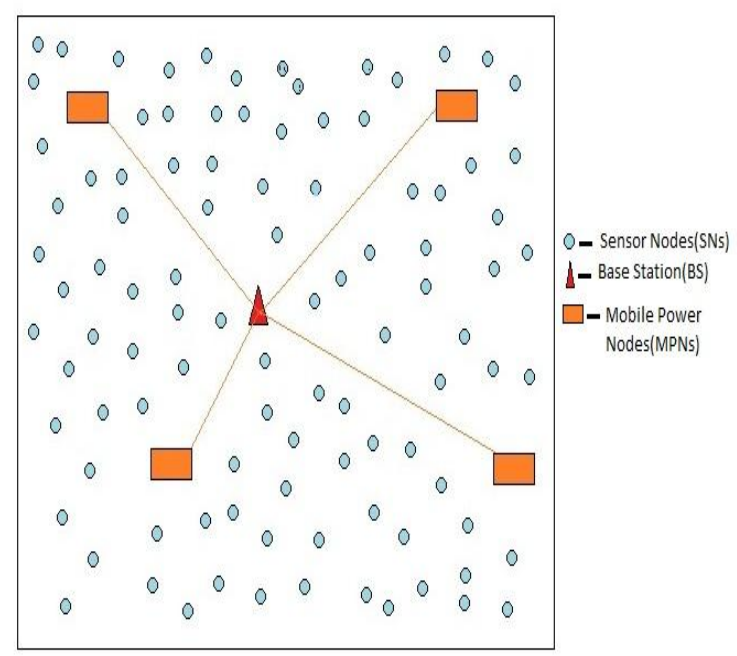

Figure 2: Deployment of Network

\section{Data Reporting Model of a SN}

The system uses the concept of geographical forwarding for the data reporting from the SN to the BS. Each SN maintains a Geographic Neighbour set i $\left(\boldsymbol{G N} \boldsymbol{S}_{\boldsymbol{i}}\right)$ which is a set of nodes in the radio range of $\boldsymbol{S} \boldsymbol{N}_{\boldsymbol{i}}$; a forwarding set of $\boldsymbol{S} \boldsymbol{N}_{\boldsymbol{i}}$ which is a set of nodes that belong to $\boldsymbol{G N} \boldsymbol{S}_{\boldsymbol{i}}$ and are closer to destination. Therefore

$F S_{i}\left(\right.$ Destination $=\left\{\right.$ node $\in G_{N} S_{i} \mid S-S_{-}$next $\left.>0\right\}$

Where $\mathbf{S}$ is the distance from $\boldsymbol{S} \boldsymbol{N}_{\boldsymbol{i}}$ to the destination i.e. base station and S_next is the distance from the next hop forwarding candidate to the destination. It is worth noticing that the membership of the neighbour set only depends on the radio range, but the membership of the GNS also depends on 
destination area. $\boldsymbol{S N _ { i }}$ chooses a next hope forwarding candidate node from one of the forwarding sets and delivers the packet to that candidate node which in turn delivers the packet to next candidate set. Finally, the packet is delivered to the BS. Our system uses the back pressure propagation to avoid the congestion in the network, if occurs.

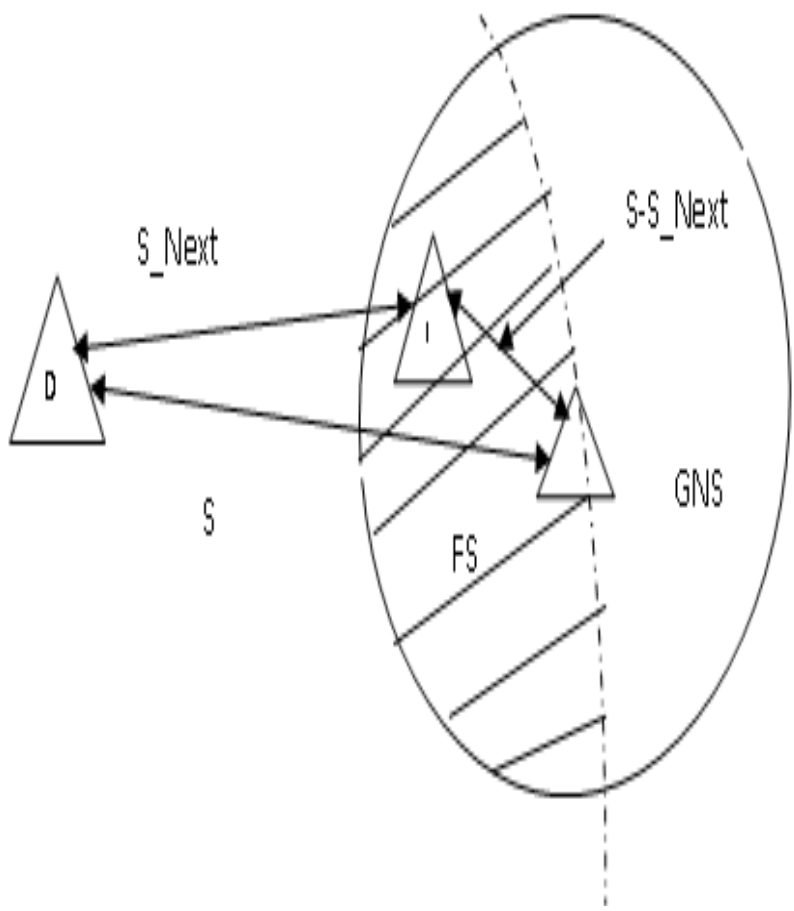

Figure 3: Geographic Data Forwarding Model

\section{Energy Dissipation Model}

For each $\boldsymbol{S} \boldsymbol{N}_{\boldsymbol{i}}$ Energy dissipated to transmit a $\mathbf{B}$ bit packet over a distance $\mathbf{S}$ to any node in neighbour set $\boldsymbol{G N} \boldsymbol{S}_{\boldsymbol{i}}$ is

$$
E_{T_{r s} s_{i}}(B, S)=B . E_{b}+B . \epsilon_{f s} * S_{t o G N S_{i}}^{2}
$$

Energy dissipated in transmitting a total of no of packets $\boldsymbol{N}_{\boldsymbol{p t c d _ { i }}}$ and receiving a total no. Of packets $\boldsymbol{N}_{\boldsymbol{r c d _ { i }}}$ is

$E_{D_{i}}=N_{p_{t c d_{i}}} * \boldsymbol{E}_{\text {Trs }_{i}}+\boldsymbol{N}_{\boldsymbol{r c d}_{\boldsymbol{i}}} * \boldsymbol{E}_{\boldsymbol{r e c}_{\boldsymbol{i}}}$

Where energy $\boldsymbol{E}_{\text {rec }_{i}}$ is the energy dissipated in receiving a message of bits B is

$\boldsymbol{E}_{\text {rec }_{i}}=\boldsymbol{B} . \boldsymbol{E}_{\boldsymbol{b}}$

Therefore energy remaining at any instant of time is will be

$E_{R_{i}}=E_{T_{i}}-E_{D_{i}}$

The energy factor Index (EFI) of a $S N_{i}$ is

$\operatorname{EFI}_{i}=E_{R_{i}} / E_{T_{i}}$

Value of $\boldsymbol{E} \boldsymbol{F} \boldsymbol{I}_{\boldsymbol{i}}$ shall be in the range of 0 to 1 . On the basis of ESI BS categorizes the energy level of each node in three ways:

Healthy $(\mathrm{H})$ : Healthy nodes are those nodes which have $\mathrm{EFI}>=0.6$.

Critical(C): Critical nodes are those nodes which have $\mathrm{EFI}<0.3$.

Affected (A): Affected nodes are those nodes with $0.3<=\mathrm{EFI}<0.6$.

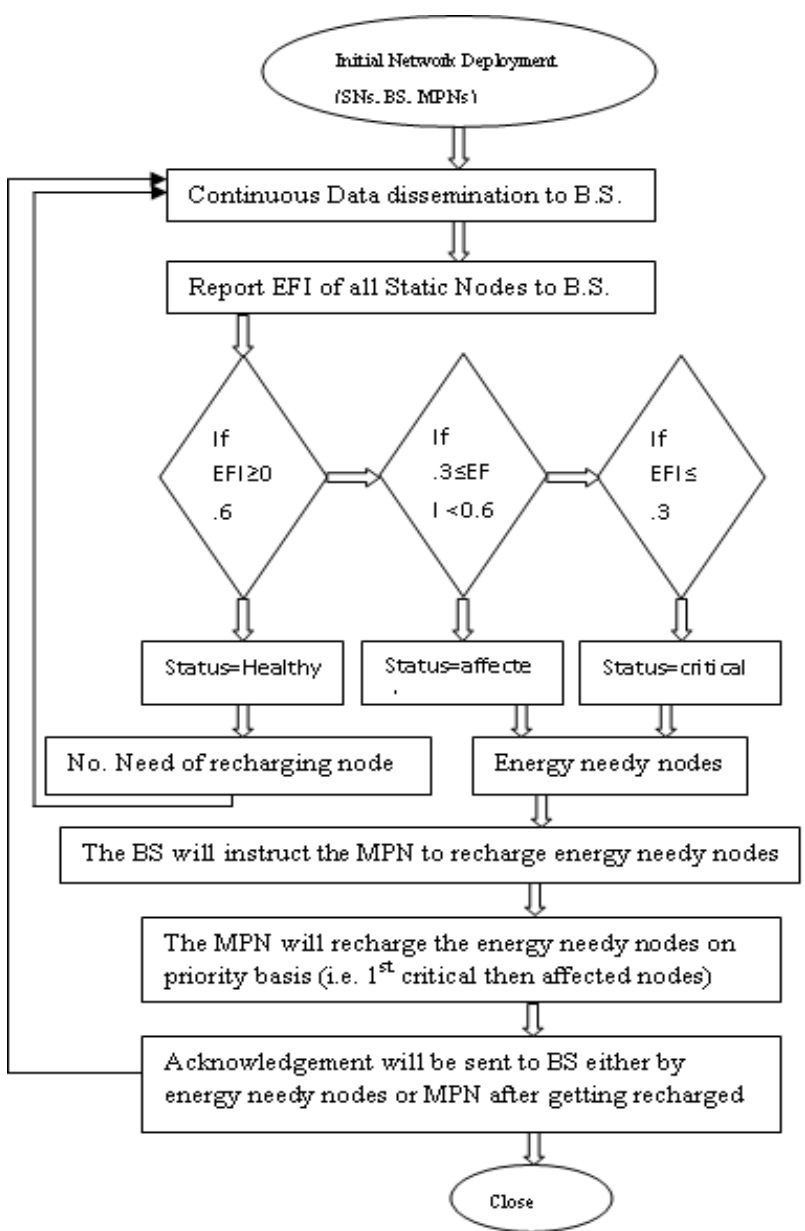

Figure 4: Flow Diagram of SCFDET in EH-WSNS

\section{Energy Transfer Algorithm}

The below mentioned algorithm is for transfer of energy from MPNs to Energy needy nodes.

\section{Energy Transfer $(N, P)$}

$N-S e t$ of all nodes in Sensor Field

$P-S e t$ of all MPNs Deployed in Sensor Field

1. If $N=\emptyset$ or $P=\varnothing$

$$
\text { Exit }
$$

2. If $i \in N \& \&$ Status $_{i}=$ Healthy Continue Operation

3. If $i \in N$ \&\& Status $_{i}=$ Affected Add $i$ to priority $Q_{A}$

4. If $i \in N$ \&\& Status $_{i}=$ Critical Add $i$ to priority $Q_{C}$

5. If $i \in P \& \& Q_{C} \neq \varnothing$ Pop the request $R_{j}$ in front of $Q_{C}$ Move the nearest_to $(J)$ to $\operatorname{loc}(J)$ Reharge $(i, j)$

6. If $i \in P \& \& Q_{A} \neq \varnothing$ Pop the request $R_{j}$ in front of $Q_{A}$ Move the nearest_to $(J)$ to $\operatorname{loc}(J)$ Reharge $(i, j)$

\section{Exit}

\section{SIMULATION RESULTS}

Table 1 represents an insight of the initial setup of the system in terms of total number of sensor nodes, MPNs, size of the network, channel bandwidth etc. 
Table 1: Characteristics of the Test Network

\begin{tabular}{|l|l|}
\hline Parameter & Value \\
\hline Number of Sensor Nodes & 100 \\
\hline Number of MPN & 04 \\
\hline Network Size & $100 \mathrm{~m} * 100 \mathrm{~m}$ \\
\hline Base Station Location & $(50,50)$ \\
\hline Channel Bandwidth & $1 \mathrm{Mb} / \mathrm{s}$ \\
\hline Radio Propagation Speed & $3 * 103 \mathrm{~m} / \mathrm{s}$ \\
\hline Initial Energy of MPN & $50 \mathrm{Joules}$ \\
\hline Initial Energy of each node & .005 Joule \\
\hline
\end{tabular}

The initial experimental deployment of the system can be observed from the figure 5. The Base station is placed in the center of the network field and fixed in nature. The network is randomly deployed. Also there are 4 MPNs deployed in the sensor field. The sensor node will perform the assigned task and send the information to the BS. The BS will receive the information from sensor nodes and forward it to the end user.

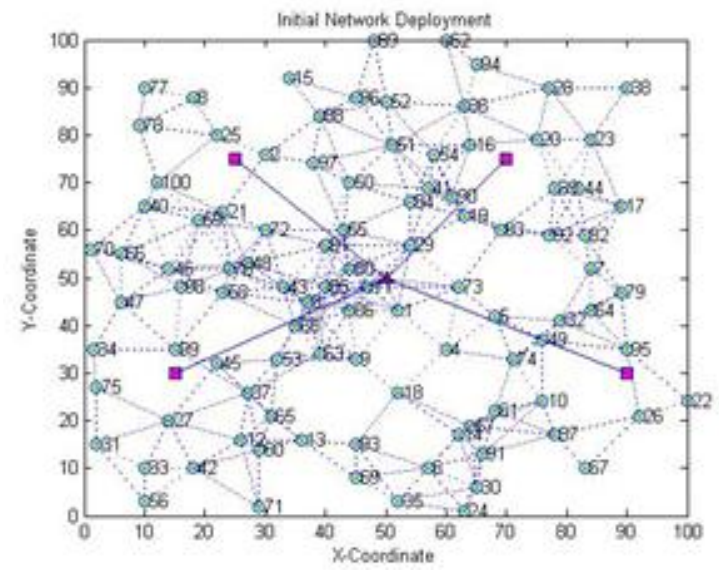

Figure 5 Initial Network Deployments

Figure 6 shows the energy level of each node after performing some round of data dissemination to the BS. Initially energy level of each node was $0.005 \mathrm{~J}$. The energy level of nodes varies along with the data dissemination to the BS. The X-axis represents the number of nodes .The $\mathrm{Y}$-axis represents the energy in terms of joule. The green line indicates energy level for the corresponding nodes.

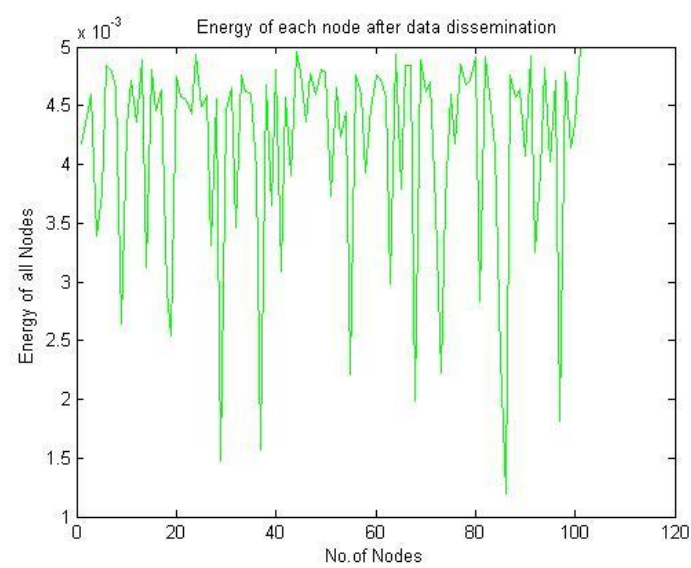

Figure 6: Energy of Each node after Data Dissemination
Figure 7 shows the energy level of Critical nodes before recharging. After performing some round of data dissemination the energy level of sensor nodes falls into Critical category. The $\mathrm{X}$-axis represents the number of nodes. The Y-axis represents Critical category energy value .The black circles represent the Critical nodes with corresponding energy.

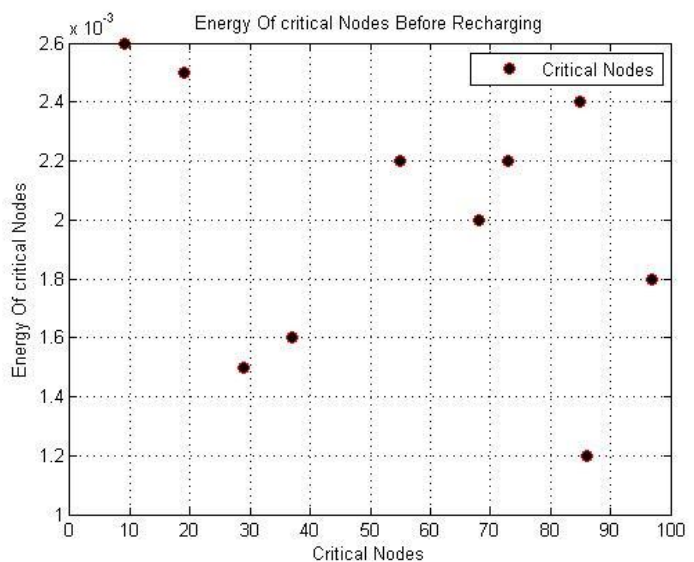

Figure 7: Energy of Critical Nodes before Recharging

Figure 8 shows the energy level of Critical nodes after recharging. After performing some round of data dissemination the energy level of sensor nodes falls into Critical category. The MPN is used to recharge the Critical category nodes. After recharging the Critical nodes; the energy level of Critical nodes falls into Healthy category. The X-axis represents the number of nodes. The Y-axis represents Healthy category energy value. The blue circles represent the Critical nodes with corresponding energy after getting recharged by the MPN.

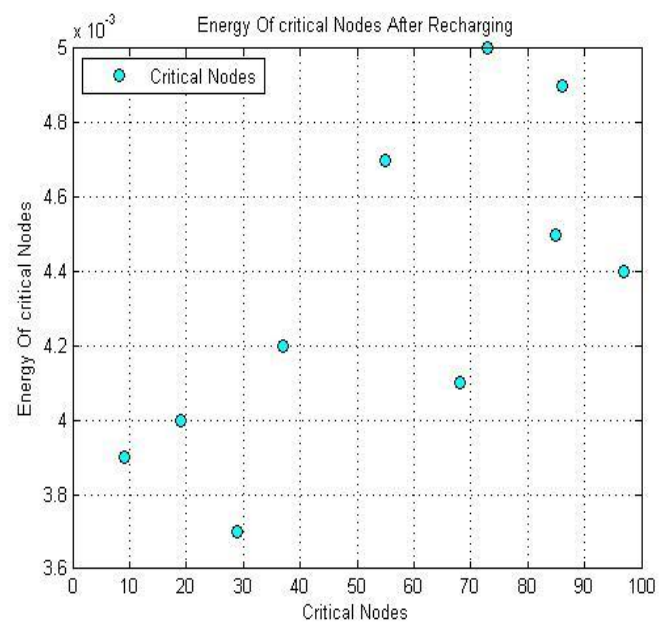

Figure 8: Energy of Critical Nodes after Recharging

Figure 9 shows the energy level of Affected nodes before recharging. After performing some round of data dissemination the energy level of sensor nodes falls into Affected category. The X-axis represents the number of nodes. The Y-axis represents Affected category energy value .The red diamonds represent the Affected nodes with corresponding energy. 


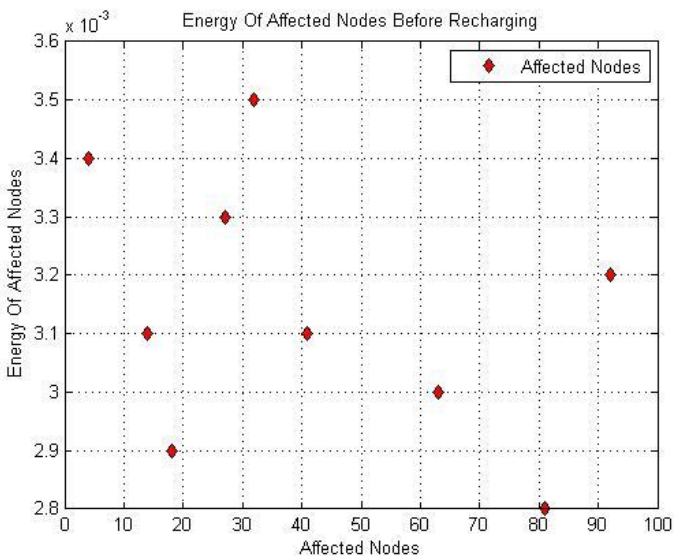

Figure 9: Energy of Affected Nodes before Recharging

Figure 10 shows the energy level of Affected nodes after recharging. After performing some round of data dissemination the energy level of sensor nodes falls into Affected category. The MPN is used to recharge the Affected category nodes. After recharging the Affected nodes; the energy level of Affected nodes falls into Healthy category. The $\mathrm{X}$-axis represents the number of nodes. The Y-axis represents Healthy category energy value .The blue diamond's represent the Affected nodes with corresponding energy after getting recharged by the MPN.

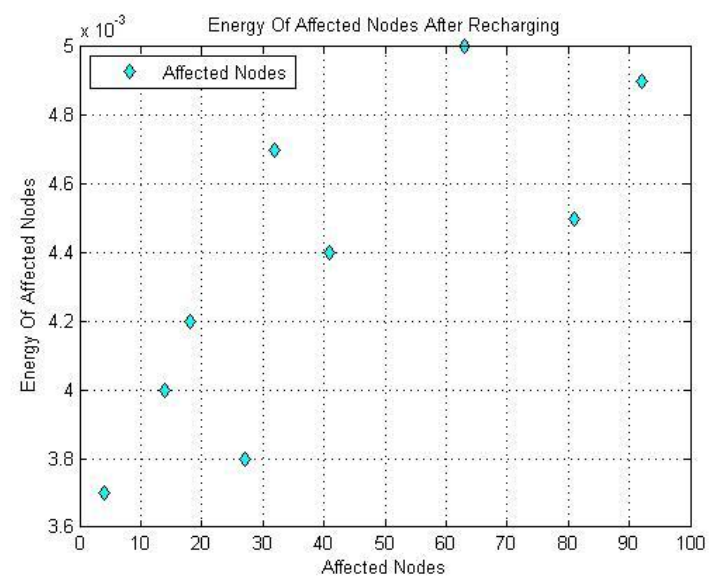

Figure 10: Energy of Affected Nodes after Recharging

\section{CONCLUSION AND FUTURE WORK}

A wireless sensor network is usually constrained by a poor network life time and poor data delivery. Power Ambulance system for wireless sensor networks is a continuous energy harvesting system which caters with the requirements of energy of the sensor nodes efficiently. Use of energy harvester and super capacitor provides the required energy density to charge and provide the on field solution to the problem. The geographic forwarding concept ensures an efficient data delivery. Backpressure approach solves the network congestion problem.

Finding an optimal strategy for the optimized movement of the MPNs in Power Ambulance System is still a field to be worked on. However a future possibility lies in providing the energy harvester for all nodes. A secure dissemination scheme is highly desired once the energy problem is solved. Not all applications have an applicability of energy harvester at all places. One may work to find an alternative to on field energy harvester or may develop an artificial energy harvester. Finding an optimal strategy for the optimized movement of the MPNs in Power Ambulance System is also a field to be worked on.

\section{REFRENCES:}

[1] S. Chalasani, J. Conrad, "A Survey of Energy Harvesting Sources for Embedded Systems", Southeastcon, 2008, IEEE, pp 442 - 447, April 2008

[2] Marcos Augusto M. Vieira, Claudionor N. Coelho. Jr, Diogenes Cecilio da silva Junior, and Jose M. da Mata, "Survey on Wireless Sensor Network Devices", Proceedings of ETFA'03, IEEE, 2003, vol. 1, pp. 537544.

[3] C. Alippi, G. Anastasi, M. Di Francesco, M. Roveri, "Energy Management in Wireless Sensor Networks with Energy-hungry Sensors", IEEE Instrumentation \& Measurement Magazine, Vol. 12, No. 2, April 2009, pp.16-23.

[4] G. Park, C.R. Farrar, M.D. Todd, W. Hodgkiss, R. Rossing, "Energy Harvesting for Structural Health Monitoring Sensor Networks", Technical Report, Los Alamost National Laboratories, LA, February 2007.

[5] Atwood B, Warneke B and Pister K S J, "Smart Dust mote forerunners," Proceedings of 14th Annual International Conference on Micro electromechanical Systems, 2001, pp 357-360.

[6] A.K Bindal, A. Mangla, D. Prasad, R. B Patel, "PLDP: Possible Location Deployment Protocol For Energy Harvesting in WSNs" , 2nd IEEE International Conference on Parallel Distributed and Grid Computing, PDGC 2012,6-8 Dec. 2012,PP 574 - 579.

[7] Michael R. Hansen, Mikkel Koefoed Jakobsen and Jan Madsen, "A Modelling Framework for Energy Harvesting Aware Wireless Sensor Networks" in Sustainable Energy Harvesting Technologies - Past, Present and Future, 2011, pp. 3-24.

[8] D. Mascarenas, E. Flynn, C. Farrar, G. Park, and M Todd, "A mobile host approach for wireless powering and interrogation of structural health monitoring sensor networks," Sensors Journal, IEEE, vol. 9, no. 12, pp. $1719-1726$, dec.2009.

[9] T. Starner, "Human-powered wearable computing", IBM System Journal, vol. 35, nos 3\&4, 1996.

[10] M. S. Halper and J. C. Ellenbogen, "Supercapacitors: A Brief Overview”, MITRE, McLean, Virginia, 2006.

[11] Amit Kumar Bindal, Nitin Jain, Anuradha Bindal, Divya Popli , "Potential Identification Scheme for Energy Harvesting Wireless Sensor Networks", 4th International Conference on Emerging Trends in Engineering and Technology (IETET-2013), "DOI 03.AETS.2013.3.156". 